doi: 10.52370/TISC21334MP

\title{
MATHEMATICAL MODELLING AND STATISTICAL ANALYSIS IN POST - COVID-19 TOURISM RECOVERY
}

\author{
Marija Paunovic ${ }^{1}$
}

\begin{abstract}
The COVID-19 pandemic, along with responses to pandemic control in the form of a national blockade with widespread application of travel restrictions, has made tourism one of the most vulnerable sectors. With increasing mitigation measures in many countries regarding tourism and travel restrictions in some segments, different tourism recovery programs are being launched according to the specifics of each country. The paper will consider various proposed measures through different impact factors set in order to accelerate the recovery of the tourism sector. Selected mathematical models will be presented and discussed.
\end{abstract}

Key Words: Mathematical modelling, Markov chain, Stringency Index, forecasting, pandemic effects, government policy

JEL classification: $C 02, C 3, C 63, D 8, Z 3$

\section{Introduction}

The outbreak of Covide-19 has affected countries in different times, in different ways and to varying degrees. The response of states was also different in terms of pandemic control and turned into national blockades and widespread application of travel restrictions and border closures, making tourism one of the hardest hit sectors. It has been shown that the impact of the crisis needs to be viewed through interconnected systems and stakeholders. The tourism sector is very sensitive to shocks such as the pervasiveness of pandemics due to a sudden decrease in demand (Chang et al., 2020). Special, the hotel industry suffers dramatic losses as the occupancy rate has fallen mainly due to social distance and a drastic drop in the number of tourists. (Sobieralski, 2020).

\footnotetext{
${ }^{1}$ Marija Paunović, PhD, Associate Professor, Faculty of Hotel Management and Tourism, University of Kragujevac, majap@ rcub.bg.ac.rs
} 
Goverment policy regarding lockdown has a direct impact on the hospitality industry. No universal procedures have been set for actions during a pandemic, but on the other hand, this problem and the impact of a pandemic have been little discussed in the literature, especialy to reduce the negative effect of a pandemic on the hospitality industry. Objectives and strategies should be purposely developed and coordinated and should not just derive from day-to-day operating decisions (Marjanović, 1999).

One of the key goals is revitalization and stabilization of economy. Stabilization includes eliminating unfavourable trends in the economy as a whole, or in the particular sector. Management must ensure that a minor external disturbance causes only a minor deviation from the equilibrium and that, once the disturbance ends, the system returns to equilibrium (Đorđević et al., 2014).

Even before the pandemic, modern working environments faced the uncertainly due to technological progress, changes in economic, and political unreability (Etehadi \& Karatepe, 2019). The influence of pandemic on the tourism sector is reflected not only in lower supply-side revenues, but also at the individual risk perception on demand-side. Security issues are a key factor in willingness of tourists to travel and strongly influence tourist demand. Until recently, tourism security experts were concerned about incidents such as physical attacks on guests, personal robberies, acts of violence, and cyber theft.

As a very complex system which is practically connected to many aspects of modern life, tourism must be observed through interdisciplinary studies (Paunović \&Vasović, 2020). Uncertainties and vagueness are the most common reasons for errors in evaluation of characteristics and values of certain occurrences, because clear and accurate information on the environment is not often available. In decision-making processes, including tourism, decisions are usually made in terms of uncertainty or absence of information or knowledge of a particular problem, so it is necessary to make different assessments and to make proper solutions to the problem (Paunović \&Vasović, 2020).

The biggest concern of the people and the government about the influence of this pandemic on people's lives and the world economy was the pandemic peak, the duration and the number of infected people. Scientific communities from different fields, and in many cases in joint cooperation, sought to contribute to the effort to cope with the current pandemic using 
the principles of applied mathematics, statistics and artificial intelligence (AI) and machine learning techniques to construct predictive models.

Tourism is among the most complex industries from the point of view of integrating, analyzing and processing data. Having in mind specific features of tourism services, the assessments are based on objective and subjective standpoints of service users. Therefore, in addition to classical mathematical and econometric models, as well as statistical analysis, the techniques of the fuzzy mathematics and the theory of uncertainty will be used for the purpose of quantification and prediction in accordance with the requirements of certain project tasks. "Soft computing techniques are a group of unique methodologies, often complement to each other, and prepare extensible knowledge handling efficiency in solving real-life issues" (Paunovic et a., 2018, p. 122). The special contribution of these techniques is in information analysis and decision making in complex systems (Paunović et al., 2020a).

In this research, the author will model influence of the government response in terms of pandemic control through Markov chains modelling and OxCGRT Stringency Index (SI) base on tracks of the individual policy measures through 19 indicators, for the short-term forecast in new Covid 19 cases in Serbia.

\section{Statistical analyses literature review}

There are many researches that have dealt with the inluence of Covid 19 on the tourism sector from different points of view. In this section, we will single out papers based on statistical tools, the results of which can help local authorities and government in policy setting.

Methods and tools of qualitative word frequency analysis words are increasingly used in various disciplines, including pandemic research and the impact on the travel industry. Methods do not generate a meaningful big picture even if they have a significant frequency, so it needs to be combined with other methods. The concept of text mining, which is accepted as a specific type of data mining, can be defined as "the process of extracting hidden information from textual data" (Feldman \& Sanger, 2007, p. 1).

The authors (Uğur \& Akbiylk, 2020) investigated pandemic travel using this method. The word analysis lists 500 words with the highest tf $*$ idf 
value and the highest frequency. Result of analyzes are words people, travel, virus, day, case, time, cancellation and travel stand out, as well as the words flight, week, coronavirus, China, post office, country and good. The highest frequency has the term Corona and then travel insurance.

Similary, term frequency analysis was done for cruise ship traveling in Pan et al. (2021). Authors investigated external factors (passenger restrictions), passenger behavior, attitudes and intentions of after pandemic demand. Pan et al. used Structural equation modeling (SEM). The attention was to assess conection between hiden variables without the influence of measurement errors. SEM was applied to test the measurement model through confirmatory factor analysis (CFA), and the hypotheses test with a structural model. Model fit was measured using $\chi 2$-distribution, comparative fit index (CFI), normalized fit index (NFI), and mean square approximation error correction. Basic statistical parameters were determined as cell statistics, and ANOVA was implemented too in this research. Substantial subjects (e.g. Covid 19, health, cleanliness) have the upmost frequency, while items related to price and costs (eg facilities, accommodation) are on the second place.

The study (Qiu et al, 2020) estimated the social costs related to residents in tourist destinations in the midst of the epidemic applying the contingent valuation $(\mathrm{CVM})$ model. In particular, the willingness of local residents to pay (WTP) to reduce risk of COVID-19 was assessed, including the aim to minimize negative effects and social costs in tourism sector. The values, curves of demand and social costs of tourism were compared applying the model of triple bounded dichotomous selection of contingent and induced WTP. One of the findings implies that younger generations want to pay more to reduce risk, so goverment together with tourism entities should include younger people, who are more motivated and more versed in postpandemic crisis recovery programs.

Ming et al. (2020) explore the influence of government measures to pandemic on the stock returns of selected tourism entities listed on the stock exchange together with Stringency Index. Conclusion points to deacrease in stock returns of those entities. Stocks of small entities with less tangibility, and more reserves are flexible to the situation and vice versa. There is a noticeable greater influence on entities from the tourism sector, airlines, as well as the casinos and similar operation. 


\section{Stringency Index}

Project The Oxford Coronavirus Government Response Tracker (OxCGRT) calculates a Government Stringency Index (SI) base on tracks of the individual policy measures through 19 indicators. The index is the average of the indicators of individual components, and the daily index is determined as an average rate of 9 indicators, each of which takes a value between 0 and 100. The authors emphasized that the index records the rigor of government policies, not the appropriateness or efficiency of the country's response. A higher value does not necessarily imply that the country's response is "better" than the other lower ones in the index. Figure 1a shows SI index, that is, Serbia's Covid-19 trajectory along with daily deaths, in the period from Jan 01, 2020 - Feb 13, 2021.

Figure 1a: The Stringency Index for Serbia

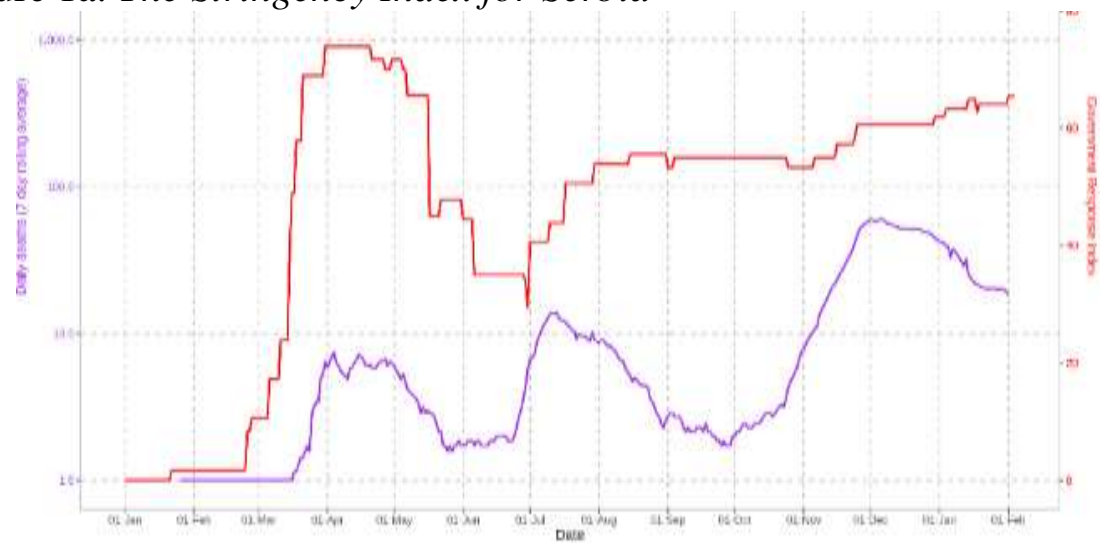

Source: The Oxford COVID-19 Government Response Tracker (OxCGRT), 2021

Figure 1b. Coronavirus Government response tracker for Serbia

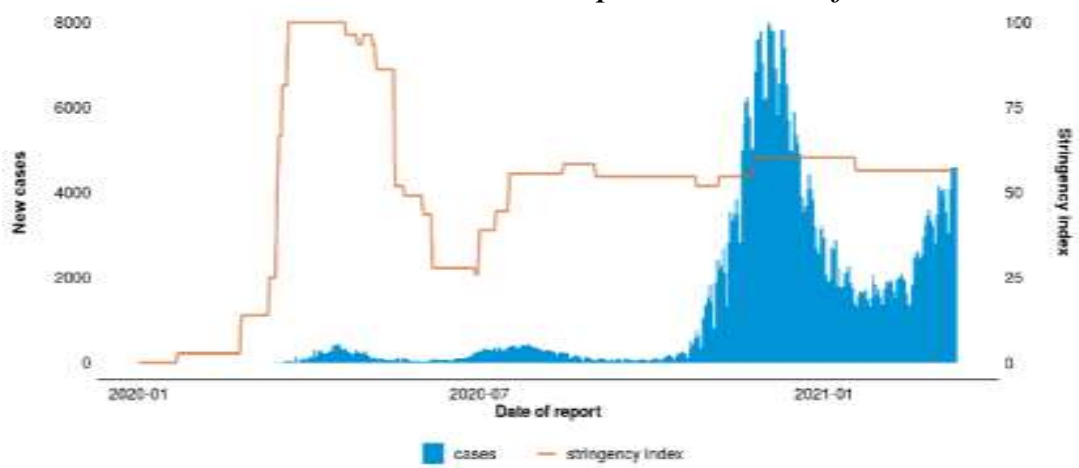

Source: The Oxford COVID-19 Government Response Tracker (2021) 
Table 1: SI index for Serbia (OxCGRT)

\begin{tabular}{|c|l|c|}
\hline No. & \multicolumn{1}{|c|}{ Policy } & Current index \\
\hline 1 & Oxford Stringency Index & 56 \\
\hline 2 & School closing & 33 \\
\hline 3 & Workplace closing & 67 \\
\hline 4 & Cancel public events & 100 \\
\hline 5 & Restrictions on gatherings & 100 \\
\hline 6 & Close public transport & 0 \\
\hline 7 & Stay at home requirements & 33 \\
\hline 8 & Restrictions on internal movement & 50 \\
\hline 9 & International travel controls & 25 \\
\hline 10 & Income support & 100 \\
\hline 11 & Debt/contract relief & 50 \\
\hline 12 & Public information campaigns & 100 \\
\hline 13 & Testing policy & 67 \\
\hline 14 & Contact tracing & 100 \\
\hline
\end{tabular}

Source: The Oxford COVID-19 Government Response Tracker (2021)

The following figure gives a comparative overview of the SI for Serbia, Romania, Hungary, Bulgaria and Croatia. It can be noticed that at the beginning of the epidemic, Serbia had the strictest measures (i.e. $100=$ strictest response), and mostly until the end of November and the beginning of December. Relaxation of measures was recorded in June. In the last few months, SI has been at a medium level compared to selected countries. More about the index and methodology can be found in OxCGRT project.

Figure 2: Comparative overview of SI by countries

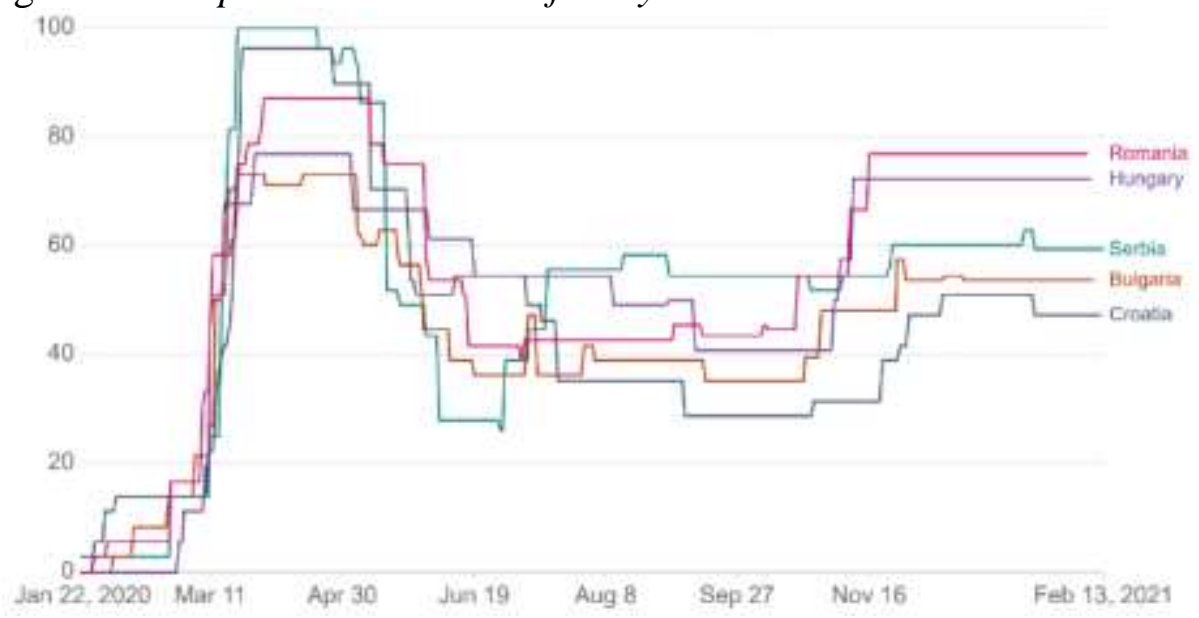

Source: The Oxford COVID-19 Government Response Tracker (2021) 
The SI index was further used in the proposed model.

\section{Mathematical modelling}

Great influence on the complex business systems have its subsystems, as well as relations between them. The availability of information and experience generated from historical data, and often constrained time period, effect the final decision of selection. Also,"some unknown and rare situations and problems may arise in practice that can neither be foreseen nor prevented, but can have impact on the work of business systems". (Paunovic et al. 2018, p. 3). Some flexible models introduced in (Gajović et al., 2018; Paunović et al., 2020b) can help to model uncertainty and can be used in different disciplines.

In decision-making processes, the decision is usually made in conditions of uncertainty, i.e. the absence of information or knowledge about a particular problem, so it proved necessary to make different assessments and find different solutions to a given problem. Uncertainty implies phenomena whose outcomes cannot be accurately predicted in advance. Uncertainty theory is a part of mathematics that, among other things, deals with modeling the degree of belief.

The fuzzy measure theory is the generalization of the classical theory of the measure. This generalization is achieved by substituting the additive axiom of classical measure with the weaker axioms of monotonicity and continuity. The development of this theory arise from the fact that the property of additive in some applications is too restrictive and, therefore, unrealistic.

Credibility measure (Liu \& Liu, 2002), as a concept for the measure of a fuzzy event, is a set function satisfying normality, monotonicity, selfduality and maximality. Generalized credibility theory is proposed by Ralević \& Paunović (2019). A new fuzzy measure is introduced, called ccredibility measure as aggregation of possibility measure and necessity measure. The generalization of this measure in fuzzy environment is achieved through the aggregation function. "Aggregation of information takes a significant place in many knowledge based systems, where aggregation of data or values is needed." (Paunović et al., 2020b, p. 131). In general, aggregation, simultaneously, using various parts of information from different sources, in order to perform a satisfactory solution. These measures are increasingly used in real life problems and will be considered 
in future research by those authors related to topics of the post Covid 19 and tourism industry.

The DEMATEL (Decision-Making Trial and Evaluation Laboratory) method is very suitable in designing and studying a structural model. This is achieved through the definition of causal relationships among complex elements (Pamučar \& Ćirović, 2015). The The model uses graph theory as a basis and enables visual planning and problem solving. Thus, the relevant elements can be splitted into causal and consequential in order to better understand the interrelationships. This achieves a better understanding of the complex composition of the considered problem, determines the relationships among factors, the relationships among the levels of composition and the strength of element's influence.

Fuzzy DEMATEL methodology was used to calculate the cause and effect relationship among the chosen indicators and to provide guidelines for decision makers to propose appropriate quarantine measures due to the COVID-19 pandemic. Findings regarding the COVID-19 pandemic effect may indicate on the hospitality industry perspective and challenges.

\section{Proposed Model for Serbia case}

An important role in the description of various events in the nature belongs to discrete Markov processes with a discrete parameter.

Definition 1. (Markov property) Future development of the process at time $\mathrm{t}+1$ depends only on state of the process at time $t$, and not on past development times. For all $\mathrm{t}=0,1,2, \ldots$ and all states $i, j, i_{t-1}, \ldots ., i_{0} \in S$ is

$$
P\left(X_{t+1}=j \mid X_{t}=i, X_{t-1}=i_{t-1}, \ldots, X_{0}=i_{0}\right)=P\left(X_{t+1}=j \mid X_{t}=i\right) \text {. }
$$

Markovian conditional probability of any future event, given any past event and the present state $\mathrm{X}$ event and depending only upon the present state. The conditional probabilities $P\left(X_{t+1}=j / X_{t}=i\right\}=p_{i j}(t, t+1)$ is transition probabilities from state $\mathrm{i}$ at time $\mathrm{t}$ to state $\mathrm{j}$ at time $\mathrm{t}+1$. The conditional probabilities $P\left(X_{t+s}=j / X_{t}=i\right\}=p_{i j}(t, t+s)$ is transition probabilities from state $i$ at time $t$ to state $\mathrm{j}$ at time $\mathrm{t}+\mathrm{s}$.

Definition 2. If $\left\{X_{t} ; t \in[0 ; \infty)\right\}$ is a discrete Markov process and if probabilities of transition $p_{i j}(t, t+s)$ do not depend on $\mathrm{t}$ and $\mathrm{t}+\mathrm{s}$, but only 
on the difference s, thus random process is called a homogenous Markov process.

We introduce the following notation:

$P(X(0)=i)=p_{i}(0)$, initial probability of state $\mathrm{i}$, $\left(p_{1}(0), p_{2}(0), \ldots, p_{n}(0)\right)=p(0)$, initial distribution of MC states, $P(X(t)=i)=p_{i}(t)$, absolute probability of state $\mathrm{i}$ at time $\mathrm{t}$, $\left(p_{1}(t), p_{2}(t), \ldots, p_{n}(t)\right)=p(t)$, absolute distribution of MC states at time $\mathrm{t}$.

Probability of transition after one step, $p_{i j}(t, t+1)$, can be write as $n \times n$ matrix called Transition Probability Matrix (TPM) as

$$
P=\left(\begin{array}{cccc}
p_{11} & p_{12} & \cdots & p_{1 n} \\
p_{21} & p_{22} & \cdots & p_{2 n} \\
\vdots & \vdots & \vdots & \vdots \\
p_{n 1} & p_{n 2} & \cdots & p_{n n}
\end{array}\right)
$$

TPM $P=\left(p_{i j}: i, j \in S\right)$ with $\mathrm{p}_{\mathrm{ij}} \geq 0$ for all $\mathrm{i}, \mathrm{j}$, is a stochastic matrix, meaning that $\mathrm{p}_{\mathrm{ij}} \geq 0$ for all $\mathrm{i}, \mathrm{j} \in \mathrm{S}$ and $\sum_{i=1}^{n} p_{i j}=1$ (i.e. each row of $\mathrm{P}$ is a distribution over S). TPM ensures a precise characteristics of the behaviour of a Markov chain. Each element in the matrix presents the probability of the transition from a particular state to the next state. The transition probabilities are commonly determined empirically, that is based solely on experiment and observation.

Given the initial distribution $\mathrm{p}(0)$, let us treat it as a row vector, and using matrix $\mathrm{P}$, we can describe dynamics of the process $\mathrm{X}(\mathrm{t})$

$$
P\left(X(0)=i_{0}, X(1)=i_{1}, \ldots, X(k)=i_{k}\right)=p_{i_{0}}(0) p_{i_{0} i_{1}} \cdots p_{i_{k-1} i_{k}}
$$

We denote transition probability from the state $i$ to the state $j$ after s steps

$$
p_{i j}^{s}=p_{i j}(t, t+s) \text { for } s=1,2, \ldots, p_{i j}^{0}= \begin{cases}0, & i \neq j \\ 1, & i=j .\end{cases}
$$

and transition probability matrix of the homogeneous Markov chain after s steps 


$$
\begin{aligned}
& P^{s}=\left(\begin{array}{cccc}
p_{11}^{s} & p_{12}^{s} & \cdots & p_{1 n}^{s} \\
p_{21}^{s} & p_{22}^{s} & \cdots & p_{2 n}^{s} \\
\vdots & \vdots & \vdots & \vdots \\
p_{n 1}^{s} & p_{n 2}^{s} & \cdots & p_{n m}^{s}
\end{array}\right) \\
& p_{i j}^{(2)}=P(X(t+2)=j \mid X(t)=i \\
& =\sum_{k=1}^{n} P(X(t+2)=j \mid X(t+1)=k, X(t)=i) P(X(t+1)=k \mid X(t)=i) \\
& =\sum_{k=1}^{n} P(X(t+2)=j \mid X(t+1)=k), P(X(t+1)=k \mid X(t)=i) \\
& =\sum_{k=1}^{n} p_{k j} p_{i k}=P^{(2)}=P^{2} .
\end{aligned}
$$

Similarly, continuing process we get the matrix $P^{(s)}=P^{s}$.

For initial vector

$$
\begin{gathered}
\left.p_{i}(t)=P(X(t)=i)=\sum_{k=1}^{n} P(X(0)=k)|P(X(t)=i)| X(0)=k\right) \\
=\sum_{k=1}^{n} p_{k}(0) p_{k i}^{t}=p(0) P^{(t)}=p(0) P^{t}
\end{gathered}
$$

Put differently, the n-step transition probability of a Markov chain is the probability that going from state $i$ to state $j$ in $n$ transitions.

In the literature the terms equilibrium, stationary, and steady state are used to mean the same thing.

In terms of long-term of the chain $\mathrm{X}(\mathrm{t})$ it is useful to determine absolute probability of states $p_{i}(t)$ for large $\mathrm{t}(t \rightarrow \infty)$, then

$\lim _{x \rightarrow \infty} p_{i k}^{(t)}=\pi_{k}$ and $\lim _{x \rightarrow \infty} p_{k}(t)=\pi_{k}, i, k \in S$ and $\pi_{1}, \pi_{2}, \ldots, \pi_{n}$, are unique solutions of $\pi_{k}=\sum_{j=1}^{n} \pi_{j} p_{j k} \wedge \sum_{j=1}^{n} \pi_{j}=1$.

Now, the stationary probability distribution vector $\pi=\left(\pi_{1}, \ldots \pi_{n}\right)$, is

$$
\Pi=\left(\begin{array}{c}
\pi \\
\pi \\
\vdots \\
\pi
\end{array}\right)=\left(\begin{array}{c}
\pi_{1}, \pi_{2}, \ldots \pi_{n} \\
\pi_{1}, \pi_{2}, \ldots \pi_{n} \\
\ldots \\
\pi_{1}, \pi_{2}, \ldots \pi_{n}
\end{array}\right)
$$

limits are the matrix form as follows

$$
\lim _{t \rightarrow \infty} p^{(t)}=\lim _{t \rightarrow \infty} p^{t}=\Pi \text { and } \lim _{t \rightarrow \infty} p(t)=\lim _{t \rightarrow \infty} p(0) P^{t}=\pi,
$$

where $\pi$ is unique solution of 


$$
\pi=\pi \mathrm{P}, \sum_{j} \pi_{j}=1
$$

If the initial probability distribution is stationary, ie. $p(0)=\pi$, then all absolute probability distributions $\mathrm{p}(\mathrm{t})$ are stationary, the chain is in the statistic equilibrium. Remarks that can be made are that a homogeneous MC is characterized by the fact that the transition probabilities and consequently also the transition matrices only depend on the size of the time increment and that for homogeneous MC one can simplify the Chapman - Kolmogorov equations to the semi group property

$$
P(s+t)=P(s) \times P(t)
$$

The forces of transition and the transition probability functions are related by Kolmogorov equations.

In our research we used Markov process and panel data techniques. Process is used on rate of confirmed cases. The prediction of the rate of cases with and without government measures in the periods of their adoption is modelled. The OxCRD data shown in the figure were used as a starting point. Predictive data in periods and without government measures were compared with actual data.

The Markov prediction process have selected input variable, data processing, classification of states, and construction of state process, state probability, state transition probability matrix and forecasting the subsequent state probability of return.

Based on new cases, index for every day is calculated as ratio

$$
I_{i, t}=\frac{N_{\text {new poscases }}}{N_{\text {newtest made }}}
$$

Index $\mathrm{r}$ for every day is calculated by using a logarithmic approximation

$$
r_{i, t}=\ln \left(\frac{P_{i, t}}{P_{i, t-1}}\right)
$$


On each day, index $\left(r_{i, t}\right)$ was compared to the index from previous day $\left(r_{i, t-1}\right)$, and then all indexes were classified in categories, i.e. intervals.

We shall mark states in the observed period with $S_{i}$ and the state-space with $S=\left(S_{1}, \ldots, S_{n}\right)$.

Probability of the state is a possibility of occurrence of various states of the system. Each state of the index was allocated with the initial probability vector by calculating relative frequencies of index in each of the stated states.

Then, the state vector is:

$$
p(i)=\left(p_{1}, p_{2}, \ldots \ldots . ., p_{n}\right) \text { for } i=1,2, \ldots . . n,
$$

where $p_{j}$ are probabilities of the state $x_{j}, j=1,2, \ldots, n$.

For the observed period $\mathrm{T}$, initial state vector is

$$
p(0)=\left(x_{1} / T, x_{2} / T, \ldots, x_{n} / T\right)
$$

Matrices of transition states are created on the basis of the previous state of the system and probabilities of the next state are defined depending on the current state of the system. Creation of vectors and matrices of transition states can enable further forecast of probabilities of the state of index in future, especially for each day (date). Vectors of current states and transition matrices are created in MS-Excel, while calculation of transition matrices is done in the software package Matlab.

Model is characterised by 5 states of the system:

$\mathrm{S} 1$ - index is less than $-20 \%$

$\mathrm{S} 2-$ index is in interval $[-20 \% ; 0)$,

S3- index is 0 or unchanged,

S4- index is in interval $(0 ; 20 \%]$

S5- index is higher than $20 \%$

Input data are taken from covid19.rs, based on data of Institute of Public Health of Serbia "Dr Milan Jovanovic Batut".

Upon observing distribution vectors, Steady state vector is

$$
\left[\begin{array}{lllll}
0.02038 & 0.08333 & 0.00086 & 0.394208 & 0.501222
\end{array}\right] \text {. }
$$


Next figures show a comparative overview of real data for new cases on Feb 132021 and result prediction obtained through the proposed model.

Figure 3a: New Covid 19 cases in Serbia by dates

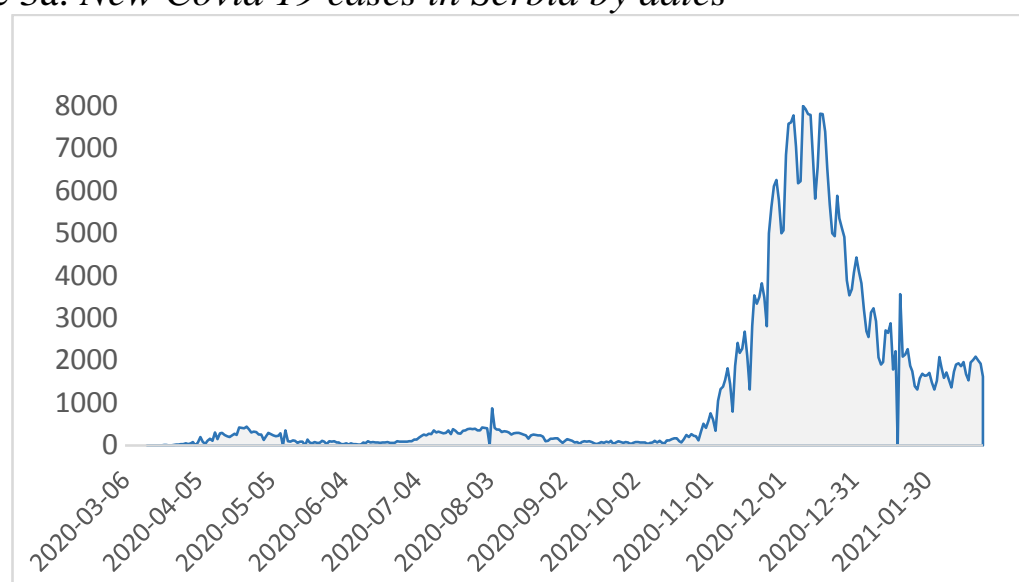

Source: Authors, based on data World Health Organization - WHO, 2021

Figure 3b: New Covid 19 cases in Serbia by dates - proposed model prediction

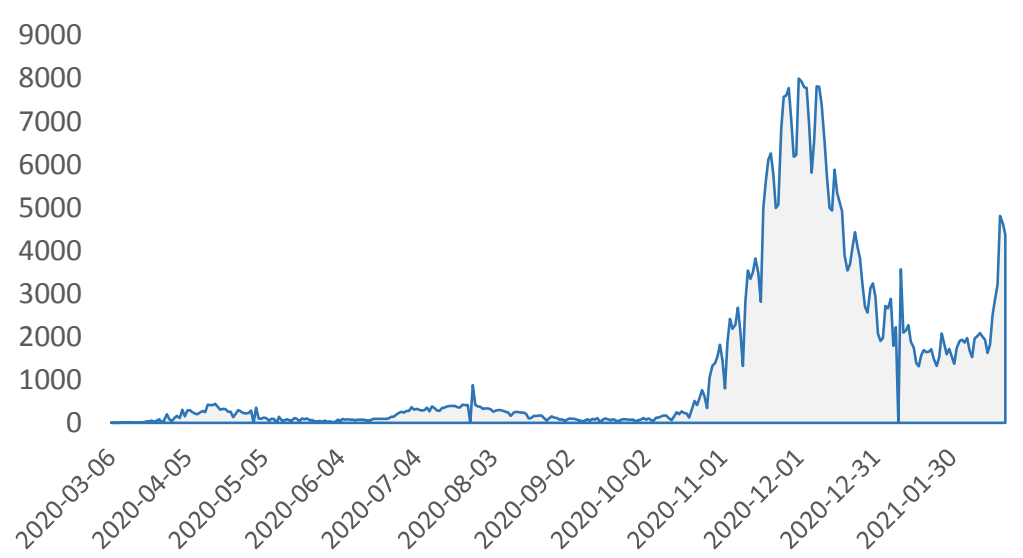

Source: Authors

Since methodology of Markov chains assumes that Markov matrix remains unchanged in time, it can be concluded that observing a long period by using this methodology is not recommended. Therefore, analysis needs to be focused on the short-term forecast, and it indicates increase in new Covid 19 cases in Serbia. 


\section{Conclusion}

According to the results of the proposed model, government measures throughout the country are yielding slow and weak results. Implementing measures to evade causing unnecessary economic damage, in some cirsumstances targeted regional blockades, may be a better solution. It is necessary to keep in mind the specifics of different industries, because the impact arising from COVID-19 also differs in industries. Therefore, policymakers need to be aware of the short-term economic consequences of restrictions. Also, conventional policy restriction may not be able to overcome this situation according to established dynamics, because people's perception of the public health risks is complex. Presented model based on Markov chains modeling and OxCGRT Stringency Index (SI) can be a guideline for short-term forecast changes in Covid 19 cases.

\section{References}

1. Altuntas, F., Gok, M.S. (2021). The effect of COVID-19 pandemic on domestic tourism: A DEMATEL method analysis on quarantine decisions, International Journal of Hospitality Management, Vol. 92, 102719.

2. Chang C.L., McAleer M., Ramos V. (2020). A charter for sustainable tourism after COVID-19. Sustainability, Vol. 12, No. 3671, 1-4.

3. Đorđević, B., I. Mihailović, I., M. Marjanović, M. (2014). The Nature of Strategic Management. International Journal of Agriculture, Inovations \& Reasearch (IJAIR), Vol. 3, No. 1, 100-120.

4. Etehadi B., Karatepe O.M. (2019). The impact of job insecurity on critical hotel employee outcomes: the mediating role of self-efficacy. Journal of Hospitality Marketing \& Management, Vol. 28, No. 6, 665-689.

5. Feldman, R., Sanger, J. (2007). The text mining handbook: Advanced approaches in analyzing unstructured data. Cambridge university press.

6. Gajović, V., Kerkez, M., Kočović, J. (2018). Modeling and simulation of logistic processes: risk assessment with a fuzzy logic technique. SAGE Journal. Simulation: Transactions of the Society for Modeling and Simulation International, Vol. 94, No. 6, 507-518. 
7. GitHub (2021). Methodology for calculating indices. https://github.com/ OxCGRT/covid-policy-tracker/blob/master/documentation/index_ methodology.md (13 February 2021)

8. Hair, J. F., Money, A. H., P, M., Samouel, P. (2007). Research methods for business. John Wiley \& Sons.

9. Liu, B., Liu, Y. K. (2002). Expected Value of Fuzzy Variable and Fuzzy Expected Value Models, IEEE Transactions on Fuzzy Systems Vol. 10, No. 2002. 445-450.

10. Marjanović, M. (1999). Biznis plan kao osnova za realizaciju menadžment strategije [Business plan as a basis for the implementation of management strategy]. Ekonomika, Niš.

11. Ming, H. C., Demir, E., García-Gómez, C. D., Zaremba, A. (2020). The impact of policy responses to COVID-19 on U.S. travel and leisure companies, Annals of Tourism Research Empirical Insights, Vol. 1, No. 1. 100003.

12. Pamučar, D., Ćirović. G. (2015). The selection of transport and handling resources in logistics centers using Multi-Attributive Border Approximation area Comparison (MABAC), Expert Systems with Applications Vol. 42, No. 6, 3016-3028.

13. Pan, T., Shu, F., Kitterlin-Lynch, M., Beckman. E. (2021). Perceptions of cruise travel during the COVID-19 pandemic: Market recovery strategies for cruise businesses in North America, Tourism Management, Vol. 85. 104275.

14. Paunović, M., Gajović, V., Ralević. M. N. (2020a). Quantitative methods for determining premium rates in transportation insurance under uncertainty, In book: Quantitative Methods in Logistics (April 6, 2020), Chapter 7, 123-138, University of Belgrade, Faculty of Transport and Traffic Engineering.

15. Paunović, M., Ralević, N., Gajović, V. (2020b). Application of the CCredibility Measure. Tehnički vjesnik, Vol. 27, No. 1, 237-242.

16. Paunović, M., Ralević, M.N., Gajović, V., Mladenović-Vojinović, B., Milutinović, O. (2018). Two-Stage Fuzzy Logic Model for Cloud Service 
Supplier Selection and Evaluation. Mathematical Problems in Engineering, Hindawi Publishing Corporation.

17. Paunović, M., Vasović, N. (2020). Evaluation model of tourism development based on uncertain linguistic information. The Fifth International Scientific Conference, Tourism in function of development of the Republic of Serbia, Tourism and Rural Development, Vrnjačka Banja 3-5 $5^{\mathrm{h}}$ September, 2020, 332-349.

18. Qiu R.T.R., Park, J., ShiNa Li, Song. H. (2020). Social costs of tourism during the COVID-19 pandemic, Annals of Tourism Research, Vol. 84. 102994.

19. Ralević N., Paunović M. (2019). c-Credibility Measure. Filomat, Vol 33, No 9, 2571-2582.

20. Sobieralski, J.B. (2020). COVID-19 and airline employment: insights from historical uncertainty shocks to the industry. TRIP, Vol. 5, No. 100123, 1-9.

21. The Oxford COVID-19 Government Response Tracker (OxCGRT) (2021). https://www.bsg.ox.ac.uk/research/research-projects/covid-19government-response-tracker (13 February 2021)

22. Uğur, N. G., Akbiyık, A. (2020). Impacts of COVID-19 on global tourism industry: A cross-regional comparison. Tourism Management Perspectives, 36, 100744.

23. Wang, J., Wang, X. (2012). Structural equation modeling: Application using mplus. Higher Education Press.

24. World Health Organization - WHO (2021). Coronavirus (COVID-19) Dashboard. https://covid19.who.int/ (13 February 2021) 